REVISIONES

Rev Obstet Ginecol Venez. 2022; 82 (1): 103-117. https://doi.org/10.51288/00820113

\title{
Microbiota de la vagina
}

\author{
José Núñez Troconis. ${ }^{1}$
}

\section{RESUMEN}

El objetivo de esta revisión narrativa fue analizar y estudiar la flora normal o microbiota vaginal, a lo largo de la vida de la mujer, así como también explicar sus diferentes mecanismos homeostáticos que permiten mantener un microambiente vaginal sano. Asimismo, mostrar los estudios de biología molecular aplicados en la microbiota vaginal, los cuales, han permitido un mejor conocimiento de la flora de la vagina. Para dicho propósito, se revisó la bibliografía latino-americana e internacional en diferentes páginas electrónicas. Se revisaron los artículos publicados desde el año 1990 hasta abril 2021. Esta revisión muestra los diferentes mecanismos que mantienen la salud $u$ homeostasis de la vaginal

Palabras clave: Microbioma, Microbiota, Microbiota Vaginal, Microflora Vaginal Normal, Lactobacilos.

\section{Vaginal microbiota}

SUMMARY

The objective of this narrative review was to analyze and study the normal vaginal flora or vaginal microbiota throughout the woman's life as well as to explain the different homeostatic mechanisms which allow a healthy microenvironment. In addition, to show the molecular biology studies used in the vaginal microbiota which have allowed to get better knowledge about the vaginal flora. For that purpose, Latin-American and international bibliographies were reviewed on different web pages. Articles published from 1991 to April 2021 were reviewed. This review shows the different mechanisms that keep the health or homeostasis of the vagina

Keywords: Microbiome, Microbiota, Vaginal microbiota, Normal Vaginal Flora, Lactobacillus.

\section{INTRODUCCIÓN}

Es bien reconocido que en el cuerpo humano coexisten colonias o comunidades o conglomerados bacterianos que son específicas para cada sitio del cuerpo, tales como el tracto gastrointestinal, la cavidad oral, la piel y la vagina. Sin embargo, el alcance de esta relación ha sido reconocido recientemente con el uso de técnicas de secuenciación de alto rendimiento (HTS). Actualmente, está claro que los microorganismos que están estrechamente relacionados con los humanos superan al total de número de las células humanas en una relación de más de 10:1, de hecho, el total de número de genes en el genoma humano es superado

${ }^{1}$ Profesor Titular. Departamento de Obstetricia y Ginecología. Facultad de Medicina. Universidad del Zulia. Maracaibo. Estado Zulia. Correo para correspondencia: jtnunezt@gmail.com

Forma de citar este artículo: Nuñez Troconis J. Microbiota de la vagina. Rev Obstet Ginecol Venez. 2022; 82(1): 103-117. https://doi. org/10.51288/00820113 por el número de genes que presenta el microbioma bacteriano en una relación superior a 1000 a $1(1,2)$.

Se denomina o se conoce como microbioma a la suma total de todas las células o microrganismos que viven en estrecha relación tanto en el interior como en la superficie exterior del cuerpo humanos y todos sus genes. El término de microbiota se refiere a la comunidad o colonia bacteriana en un sitio u órgano específico del cuerpo humano y se ha determinado por los estudios de 16s RNA realizados con técnicas de HTS. Los microorganismos y sus comunidades, colonias o conglomerados han evolucionado con los humanos por más de miles de años. Es ahora que se están comenzando a entender los complejos mecanismos que permiten conocer los beneficios de esta interacción, pero también las alteraciones de estos microbiomas pueden originar enfermedades, lo que ha mejorado los conocimientos sobre esta relación y tener un mejor conocimiento y entendimiento de las enfermedades y la salud humana (2). 


\section{J NÚÑEZ TROCONIS}

El microbioma humano mantiene una relación simbiótica con el huésped que puede comportarse como: 1.- comensalismo: no afecta al huésped; 2.- mutualismo: puede afectar positivamente al huésped y 3.- patogénico: afecta negativamente el funcionamiento, la inmunidad y el suministro de nutrientes al huésped. El microbioma humano que se adquiere durante el parto cambia durante el transcurso de la vida del huésped, especialmente el de la vagina $(1,2)$.

Recientes descubrimientos han demostrado el potencial de la microbiología molecular que ha permitido revelar características hasta entonces desconocidas del microbioma humano que son de gran importancia para la salud del humano. Los estudios moleculares han demostrado lo inadecuado de algunos métodos clásicos usados en bacteriología y han permitido demostrar que menos del $10 \%$ de los microorganismos del microbioma humano no son cultivables por las técnicas tradicionales, según la localización anatómica del microbioma. Este cambia desde el nacimiento a través de los años de la vida del humano, por ejemplo, en la vagina existe una estrecha cooperación entre los microbios con el huésped permitiendo una primera línea de defensa contra los microorganismo oportunistas, este balance es denominado eubiosis, pero cuando estos microorganismos oportunistas sobrepasan o alteran este balance simbiótico se produce lo denominado disbiosis, conllevando a una proceso inflamatorio $(1,3)$.

\section{MÉTODOS}

Se realizó la búsqueda en las páginas electrónicas de Pub Med, Google Scholar, Springer, Web of Knowledge, DOAJ (Directory of Open Access Journals), Hinari, Oxford Academic, JAMA (Journal of the American Medical Association) Network, Embase y Research Life en la literatura de habla inglesa, y en Scielo, Lantidex, Imbiomed-L, Redalyc y Google Scholar en la literatura de habla española. Se usaron y buscaron empleando los términos: microbioma, microbiota, microbiología de la vagina, microbiota de la vagina, microbioma de la vagina, flora bacteriana de la vagina, microflora vaginal normal. La búsqueda se realizó usando palabras solas o usando la combinación de $A N D / Y$ o $O R / O$. Dentro de los criterios de inclusión se consideraron: a) artículos de fuentes primarias publicados en revistas indexadas, con naturaleza de revisión, artículos originales de investigación, estudios comparativos, estudios de evaluación, capítulos de libros y metaanálisis de acceso abierto; b) artículos en idioma inglés y español. Fueron excluidos de la revisión: cartas al editor, reportes de casos y estudios sin control. Igualmente, fueron excluidas las publicaciones que no tenían libre acceso. Se revisaron los artículos publicados desde el año 1990 hasta abril 2021. Sin embargo, se incluyeron 2 trabajos, uno de la década de los 60 s y otro de la década de los 70 s que se consideraron importantes para esta revisión narrativa, asimismo, se incluyeron artículos relevantes que fueron hallados posterior a la revisión inicial.

\section{Microbiota de la vagina}

El tracto genital femenino está constituido por una sucesión de cavidades: trompas de Falopío, cavidad uterina, endocérvix y vagina, las cuales se comunican con el exterior a través del introito vulvar; estas estructuras anatómicas permiten la salida o exteriorización del flujo menstrual y el paso del feto al momento del parto, asimismo, permiten el acto sexual, pero también permiten la entrada de microorganismos potencialmente patógenos que pueden llegar a producir infecciones en el tracto genital femenino.

La microbiota o microflora vaginal (MBV), sin duda alguna, representa uno de los mecanismos de defensa más importante para mantener la función reproductiva en perfectas condiciones ya que mantiene un ambiente saludable y previene la proliferación de 
microorganismos potencialmente patógenos en la vagina $(1,2,4)$.

\section{Historia}

Anton Van Leeuwenhoek, padre de la microbiología e inventor del microscopio, fue el primero en observar una colonia o conglomerado o comunidad bacteriana humana cuando usó como muestra un raspado de sus dientes. A comienzo del siglo XIX, se añadieron estudios de numerosos investigadores de la época a través de técnicas de cultivo aeróbicos y anaeróbicos que permitieron estudiar el microbioma humano, así como también, el incremento en el conocimiento de dicha área (4).

En 1892, Albert Döderlein fue el primero en describir la importancia de las bacterias en la vagina de la mujer, las cuales, producen ácido láctico (AL), constituyendo la flora vaginal normal y siendo responsables de la inhibición de bacterias patógenas como las anaeróbicas facultativas. Pero fue Krönig, 1895, compañero de trabajo de Döderlein, el primero en diferenciar los lactobacilos de las bacterias anaeróbicas. El lactobacilo fue cultivado por Curtis en 1913 y fue llamado posteriormente Mobiluncus curtisii, por Spiegel y Roberts, en 1984, pero subsecuentemente el nombre Lactobacillus acidophilus fue propuesto para dicha bacteria (4).

A principio del siglo XX, Manu, en Finlandia, estudió y describió la flora vaginal en la mujer embarazada y no embarazada, así como también, la flora vaginal desde el nacimiento de la niña hasta la senectud. Robert Schröder, en 1921, describió 3 tipos de flora bacteriana en vagina: 1.- flora vaginal normal; 2.vaginosis bacteriana (VB); 3.- otra flora bacteriana. Luego, en 1930, Ludwig Nürnberger coincidió con la opinión de Döderlein en que solo hay 2 tipos de flora vaginal: normal y anormal (4).
En años recientes, la aparición de nuevas tecnologías moleculares y genéticas han permitido un estudio más profundo de las bacterias, incluyendo el lactobacilo, así como también, las interacciones entre ellas y con su biofilms, asimismo, determinar las diferencias genéticas de los diversos tipos de microorganismos de la flora vaginal. Antes de estas técnicas, se sabía que existían microorganismos que no podían ser cultivados ex vivo, por lo que parte del microbioma humano permanecía oscuro. El advenimiento de las técnicas de amplificación, clonización y secuenciación de los genes bacterianos usando la técnica de amplificación del ARN mensajero 16S bacteriano (rRNA 16S) en muestras de secreción vaginal, ha permitido un estudio más profundo y adecuado de los lactobacilos y de todas las especies microbianas presente en la muestra. Esto ha facilitado una mejor y más exacta descripción del microbioma humano y de la MBV. Igualmente, estas técnicas han permitido determinar que el Lactobacilo sp. (LB) no siempre es la especie dominante en la $\operatorname{MBV}(5,6)$.

Los estudios moleculares han demostrado las insuficiencias de los métodos de la bacteriología clásica $\mathrm{y}$, como se mencionó anteriormente, estas técnicas han permitido reconocer, dependiendo del lugar anatómico estudiado, que menos del $10 \%$ de los microorganismos que forman parte del microbioma humano no son cultivables. Igualmente, estas técnicas de microbiología molecular han demostrado ser potencialmente útiles en revelar características escondidas o desconocidas del microbioma humano, y por supuesto de la microbiota vaginal, así como también su impacto en la salud (2).

Uno de los sitios anatómicos más importantes para estudiar su microbiota es la vagina, en parte porque su microambiente bacteriano ha sido estudiado ampliamente usando las técnicas clásicas de microbiología y ha permitido establecer o clarificar las diferentes colonias, conglomerados o comunidades de 
bacterias que colonizan la vagina y sus interacciones, que permiten mantener una vagina sin patología o infección.

Existe una relación mutua entre la fisiología vaginal de la mujer y la MBV, inclusive con los cambios fisiológicos $\mathrm{u}$ hormonales que ocurren durante el ciclo menstrual, desde el nacimiento hasta la posmenopausia; esto significa que la composición de la microflora vaginal no es constante y presenta modificaciones o variaciones en respuesta a factores exógenos y endógenos (5, 7 - 9); estos factores incluyen las diferentes etapas del ciclo menstrual, menstruación, embarazo, uso de anticonceptivos orales, frecuencia de relaciones sexuales, uso de duchas y desodorantes vaginales, uso o ingestión de antibióticos y medicamentos con propiedades inmunosupresivas $(1,3,5,9-11)$.

La composición y estructura de la $\mathrm{MBV}$ ha sido estudiada y descrita muy bien desde el uso del microscopio de luz hasta hoy día con el uso de las técnicas de secuenciación. Se han descrito más de 250 especies de bacterias en la MBV usando la HTS, entre ellas se incluyen (orden alfabético): Actinomyces, Aerococcus, Allisonella, Alloscardovia, Anaerococcus, Arcanobacterium, Atopobium, Bacteroides, Balneimonas, Bifidobacterium, Blastococcus, Blautia,Bulleidia, Campylobacter, Citrobacter, Coriobacteriacea, Corynebacterium, Enterobacter, Escherichia, Facklamia, Faecalibacterium, Finegoldia, Gardnerella, Gemella, Haemophilus, Lachnospiracea, Massilia, Megasphera, Mobiluncus, Mollicutes, Moryella, Olsinella, Parvimonas, Peptinophilus, Peptostreptococcus, Prevotella, Porphyromonas, Proteobacteria, Providencia, Rhizobialis, Ruminococcaceae, Salmonella, Shigella, Shuttleworthia, Sneathia, Solobacterium, Staphylococcus, Streptococcus, Veillonella, Ureaplasma, y lactobacilli sp. (4, 12). En la tabla 1 , se pueden observar los microorganismos más frecuentemente encontrados o aislados en vagina $\mathrm{y}$
Tabla 1. Microorganismos frecuentes en la vagina de mujeres sanas

\begin{tabular}{ll}
\hline $\begin{array}{l}\text { Cocos y bacilos Gram positivos } \\
\text { anaerobios aerotolerantes }\end{array}$ & $\begin{array}{l}\text { Lactobacillus } \\
\text { Streptococcus }\end{array}$ \\
Cocos y bacilos Gram positivos & Corynebacterium \\
anaerobios facultativos & $\begin{array}{l}\text { Gardnerella } \\
\text { Staphylococcus } \\
\text { (especialmente }\end{array}$ \\
& S. epidermidis) \\
& Escherichia \\
Bacilos Gram negativos & Klebsiella \\
anaerobios facultativos & Proteus \\
& Mycoplasma \\
Micoplasmas & (sobre todo M. hominis) \\
& Ureaplasma \\
Bacilos y cocos Gram positivos & Atopobium \\
anaerobios estrictos & Peptococcus \\
& Peptostreptococcus \\
& Clostridium \\
& Bifidobacterium \\
& Propionibacterium \\
Bacilos Gram negativos & Eubacterium \\
anaerobios estrictos & Bacteroides \\
\hline
\end{tabular}

aislados por procedimientos convencionales. Las técnicas moleculares de secuenciación o HTS han permitido la detección de bacterias incultivables, que previamente no habían sido reconocidas o aisladas por las técnicas convencionales, originando el establecimiento de tipos de colonias o comunidades microbianas únicas (CSTs). Sobre la base de la abundancia y composición de las diferentes especies bacterianas que colonizan la vagina durante la etapa reproductiva de la mujer se han clasificado en 5 tipos: CSTI al CST-V $(1,10,13)$. La CST-I, la CST-II, la CST-III y la CST-V se caracterizan por la abundancia de los L. crispatus, L. gassei, L. iners, y el L. jensenii, respectivamente (14). Sin embargo, la CST-IV se caracteriza por estar compuesta por una mezcla de microorganismos facultativos anaeróbicos con bajos niveles de LB. sp. La CST-IV ha sido dividida en CST-IV-A y CST-IV-B. La CST-IV-A agrupa a las especies de los géneros: Anaerococus, Peptoniphilus, 
Corynebacterium, Provotella, Finegoldia y Sptreptococcus. La CST-IV-B agrupa: Atopobium, Gardnerella, Sneathia, Molbiluncus, Magsphera y Clostridiales (1, 10, 13, 14). Kalia y cols. (1) mencionan que es debatible si la CST-V representa un estado normal o un estado asintomático de una vaginosis bacteriana. Sin embargo, algunos autores (1, 10) consideran que la composición de las CSTs no es el único indicador de disbiosis.

\section{Microbiota vaginal a través de la vida de la mujer}

La mucosa vaginal actúa como un protector del tracto genital bajo contra patógenos, incluyendo el virus de inmunodeficiencia adquirida (VIH). La vagina produce una proteína surfactante A (SP-A) que provee la defensa natural a la vagina, produciendo opsonización de los microorganismos patógenos, altera los niveles de citocinas proinflamatorias, estimula los procesos oxidativos al ocasionar fagocitosis celular y estimula la diferenciación de antígenos presentados a las células; esta inmunidad adaptativa está vinculada con la inmunidad innata (15). Diferentes estudios han demostrado que la composición de la MBV también contribuye a las propiedades defensivas de la mucosa vaginal, tal como el $L$. crispatus que las incrementa o fortalece y el $L$. iners que las debilita $(16,17)$.

Como se mencionó previamente, la composición de la MBV cambia durante la vida de la mujer desde el mismo nacimiento; se ha sugerido que los cambios de la estructura del epitelio vaginal y los cambios hormonales juegan un papel fundamental en ello (1, 3, 10, 11) (Figura 1)

\section{Al nacimiento}

La colonización inicial de la vagina por los microorganismos se lleva a cabo desde la vagina materna al momento del parto o de las manos de la $1^{\mathrm{a}}$ persona que sostiene a la niña durante la cesárea (18).
Wylie y Henderson (19) refieren que el LB aparece en la vagina de la niña recién nacida a las 24-48 horas del nacimiento. Se ha sugerido que dentro de las primeras semanas desde el nacimiento, los microorganismos son capaces de diferenciarse en colonias o comunidades tanto en vagina como en los intestinos y la piel (18). Durante las primeras semanas de edad, la niña posee estrógenos prestados de la madre, lo que permite la proliferación y maduración del epitelio vaginal más la presencia de los LB, favorece la síntesis de AL (20). Al momento de su nacimiento, una niña a término tiene la vagina impregnada de los estrógenos prestados de la madre y se pueden encontrar cambios parecidos a los que se encuentran cuando la niña entra en la pubertad, durante la edad adulta y la etapa fértil, es decir, con notable desarrollo del epitelio vaginal. Se distinguen las 4 capas y presenta un grosor de 500 a $1000 \mu$, la mayor parte está compuesta por la capa intermedia bastante hiperplásica, compuesta hasta por 25 hileras de células intermedias. Por supuesto, en la niña recién nacida, se consiguen cantidades importantes de glucógeno, por acción de los estrógenos prestados de la madre hasta la 3 a 4 semana de nacida, cuando comienzan a bajar o desaparecer los niveles estrogénicos prestados (19). Estos cambios, tanto anatómicos, fisiológicos y bioquímicos, que se pueden observar en la niña recién nacida, son transitorios y desaparecen al ser metabolizados y eliminados los estrógenos prestados de la madre. Entonces, hacia el $2^{\circ}$ al $4^{\circ}$ día de nacida, la descamación celular se incrementa, asimismo, a medida que se van metabolizando los estrógenos prestados, el epitelio se va reduciendo progresivamente, y para el día $14^{\circ}$, el epitelio solo presenta unas pocas hileras de células basales.

\section{Antes de la pubertad}

La MBV durante la niñez presenta una CST-IV, un $\mathrm{pH}$ neutro o elevado y una mucosa plana estratificada fina con un epitelio delgado con solamente de 3 a 8 hileras de células a predominio de células basales y parabasales. En la niña antes de la entrada a la 


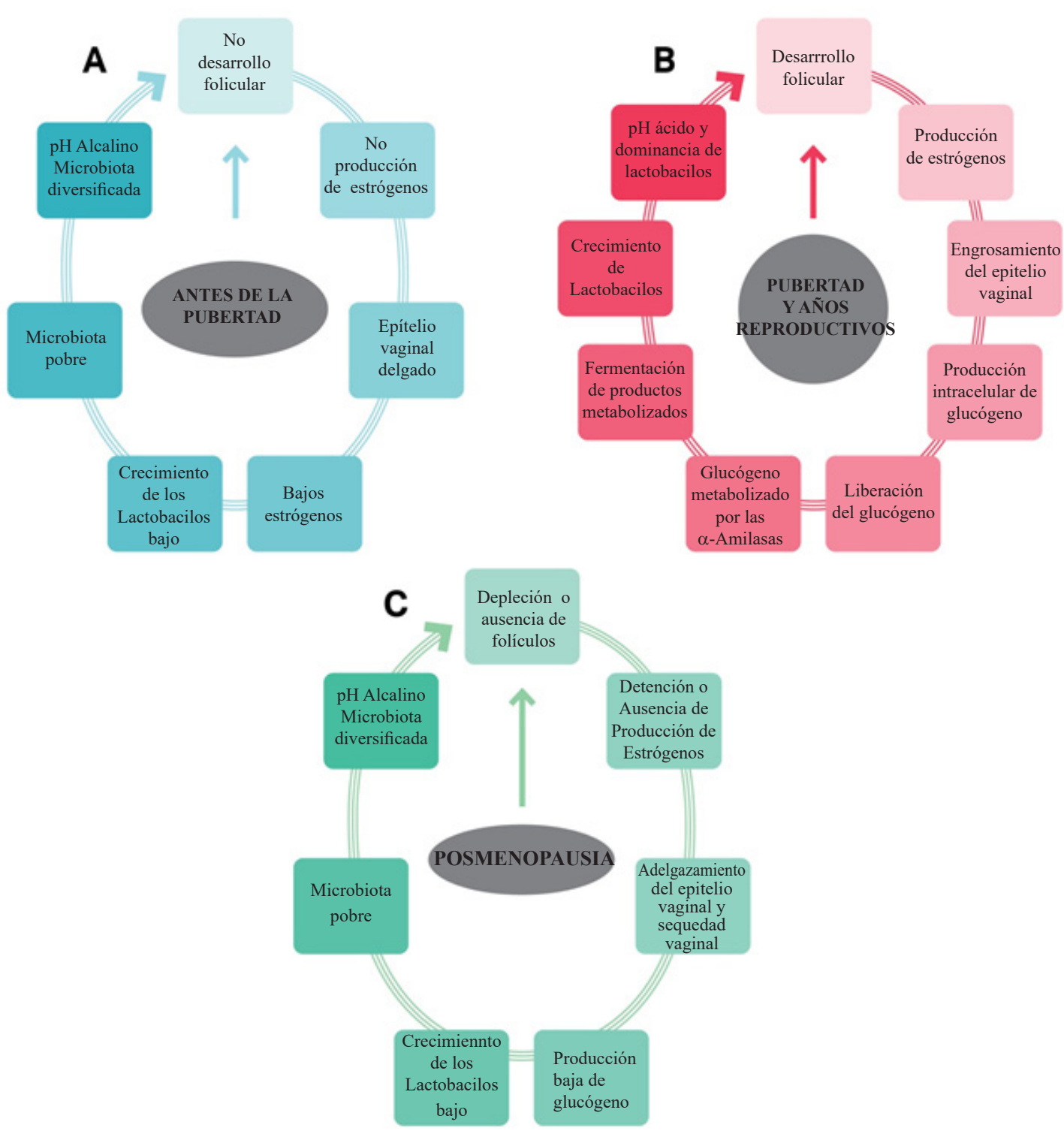

Figura 1. Microbiota vaginal a través de la vida de la mujer. a.- Niñez/años prepuberales; b.- Pubertad y años reproductivos; c.- Menopausia y Posmenopausia. Adaptado de: Kalia y cols. (1)

pubertad, los ovarios están en reposo o sea no producen estrógenos, originando mucosa muy delgada y la falta de producción de glucógeno, por consiguiente, un crecimiento bajo de los $\mathrm{LB}$, lo que produce un $\mathrm{pH}$ neutro o alcalino en la vagina (21).

\section{Pubertad y edad reproductiva}

Con la llegada de la pubertad comienza el desarrollo folicular y por lo tanto la producción de los estrógenos (Es). Niveles elevados de Es estimulan la proliferación 
y engrosamiento del epitelio vaginal con aumento de la producción de glucógeno intracelular (3). La descamación y la citólisis de las células del epitelio vaginal promocionada por los cambios hormonales, permite disponer de glucógeno libre en vagina, el cual, es procesado por la $\alpha$-amilasa presente en la vagina (22); el glucógeno y sus productos del metabolismo, tales como la maltosa y maltotriosa son fermentados por los $\mathrm{LB}$, produciéndose $\mathrm{AL}$, con la consecuente acidificación de la vagina y la dominación bacteriana de los LB en la MBV (CST I, II, II y V) con excepción de la CST-IV, que se puede encontrar en algunas mujeres $(3,23)$. Como se mencionó anteriormente, durante las fases del ciclo menstrual, el efecto de las hormonas, estradiol (E2) y progesterona (P), produce cambios en el epitelio vaginal que se manifiestan en el microambiente vaginal, como es la promoción del crecimiento de los lactobacilos. Los estrógenos estimulan el crecimiento y maduración del epitelio vaginal, es decir, las células intermedias y superficiales, y estimula la síntesis del glucógeno intracelular en estas células. La P promueve la citólisis de las células epiteliales de la vagina. Estas células, ricas en glucógeno, liberan el glucógeno que es metabolizado por los LB, así como otras bacterias que utilizan la glucosa y la maltosa, y producen AL que, como se mencionó previamente, produce y mantiene el $\mathrm{pH}$ ácido a la vagina. Diferentes estudios han demostrado que la MBV varía de una a otra mujer $(24,25)$. Estos estudios han demostrado que en algunas mujeres la MBV cambia de una CST a otra en un periodo corto de tiempo, mientras en otras, la MBV permanece relativamente constante, es decir, sin modificaciones o cambios con dominio sobre todo del L. crispatus (10, 13). Estos cambios de CST parecen estar provocados por la menstruación $(10,11)$. En el $81 \%$ de los ciclos menstruales, los niveles de la Gardnetrella vaginalis (Gv) junto al $L$. iners aumentan significativamente con la menstruación y disminuyen cuando se acerca el final de ella, mientras que los niveles del $L$. crispatus y L. jensenii se comportan en forma opuesta (11). Esto puede ser atribuido a la disponibilidad de hierro durante la menstruación, favoreciendo el crecimiento de la Gv y del L. iners. A través de los años de la mujer, mientras los ovarios estén produciendo Es, los niveles de glucógeno se mantendrán elevados, estables hasta que se alcance la menopausia $(9,26)$

\section{Menopausia y posmenopausia}

A medida que los folículos ováricos se van agotando, la menopausia se va manifestando hormonal, bioquímica, anatómica e histológicamente, se observan los efectos sobre el epitelio de la mucosa vaginal. Como consecuencia del agotamiento de los folículos, la síntesis de los Es comienza a afectarse y descender sus niveles, lo que provoca adelgazamiento del grosor de la mucosa por la disminución del número de hileras de células de las diferentes capas del epitelio plano estratificado, asimismo, se acompaña de la disminución de los niveles de glucógeno y, con ello, de los niveles de los LB que conlleva a disminución de los niveles de AL y elevación del $\mathrm{pH}$ vaginal. En la posmenopausia se observa la mucosa vaginal con características similares a la etapa prepuberal; la MBV a predominio del LB cambia a una diversidad microbiana o sea a CST-IV con reducción de las secreciones vaginales, sequedad, elevación del $\mathrm{pH}$ y por consiguiente se produce la dispaurenia. En otras palabras, la MVB de la mujer menopáusica y posmenopáusica se asemeja a la MBV de la etapa prepuberal, lo que sugiere que la fisiología reproductiva juega un papel fundamental en las características de la MBV de la mujer en sus años reproductivos $(27)$

\section{Los lactobacilos vaginales}

Desde el primer estudio microbiológico de la vagina humana, realizado por Döderlein, los LB han sido descritos consistentemente como los microbios dominantes en ella. Por ello, se considera que tienen un papel crítico en el mantenimiento del ecosistema vaginal al prevenir la excesiva proliferación de microorganismos indeseables tales como la Gv, asimismo, impide la colonización por 


\section{J NÚÑEZ TROCONIS}

microorganismos patógenos, generadores de patología urogenital (2). También es cierto que la vagina puede ser colonizada por otras bacterias alternativas como Atopobium por lo que una MBV dominada por los LB no es un requerimiento absoluto $(28,29)$, sin embargo, la presencia de esos microorganismos es esporádica por lo que se sigue considerando la presencia de los LB como fundamental para mantener la homeostasis de la vagina o sea la MBV (30).

Los lactobacilos presentan varias formas, desde muy alargadas a muy cortas, desde rectos hasta curvos e incluso formas espiriladas. Se les incluye habitualmente en el grupo amplio y heterogéneo de las bacterias productoras de AL, caracterizadas por ser grampositivas, no esporuladas y con un catabolismo de los azúcares estrictamente fermentativo, cuyo producto final predominante es dicho ácido orgánico. El AL es producido en una cantidad suficiente para mantener el $\mathrm{pH}$ de la vagina en nivel ácido, entre 3,5 a 4,5. En general, los lactobacilos son bacterias anaeróbicas tolerantes del medio aeróbico y presentan un genoma pequeño por lo que son muy exigentes desde el punto de vista nutricional.

La identificación de la especie de lactobacilo de la vagina va a depender del método empleado; se puede observar una disparidad en los hallazgos cuando se emplean métodos que identifican las características fenotípicas o métodos que identifican características genotípicas. La disparidad entre los métodos de identificación fenotípica (cultivos y aislamiento) y genotípicos (HTS/16s RNA) parece deberse a la diversidad bioquímica intraespecie de los lactobacilos vaginales y a la ausencia de algunas especies en la base de datos.

\section{Taxonomía}

La adscripción precisa de los lactobacilos aislados de vagina a especies concretas va a depender de los métodos de identificación empleados. En los métodos dependientes de cultivo se incluyen habitualmente dentro del género Lactobacillus los aislados bacilares, no esporulados, grampositivos y catalasa negativos. Adicionalmente, puede emplearse como criterio el desarrollo en medio de cultivo MRS (Man, Rogosa, Sharpe), aunque esta última exigencia puede dejar fuera a algunas especies como L. iners. En todo caso, en los aislamientos primarios, los lactobacilos se comportan como organismos fastidiosos, y es aconsejable la incubación en medios enriquecidos con hemo (hemina, hemoglobina o sangre) y la atmósfera enriquecida en $\mathrm{CO}_{2}$ o incluso anaeróbica. Una vez purificados, los LB aislados se suelen someter a las pruebas de identificación que, tradicionalmente, consisten en la determinación de la capacidad de la cepa en fermentar diferentes fuentes de carbono y de crecer en medios con concentraciones crecientes de sal $(2,18)$. Más recientemente, se han utilizado las galerías miniaturizadas y tanto estas pruebas como las otras indican que la vagina está colonizada preferentemente por L. acidophilus $(18,31,32)$ y/o L. fermentum (33). Sin embargo, cuando se aplican métodos genotípicos de identificación a los mismos gérmenes, ninguno puede adscribirse a cualquiera de esas dos especies y las predominantes son $L$. crispatus, $L$. gasseri y $L$. jensenii, tanto si se analiza el grado de homología de los ADN cromosómicos $(34,35)$ al determinar la huella genética (DNA fingerprinting) (36) o las secuencias de los ARNr 16S $(28,37)$. Por medio de estos métodos también se detectan en la vagina otras especies como L. iners $(27,38)$ y L. vaginalis $(32)$. Esta disparidad entre los métodos fenotípicos y genotípicos, como se mencionó anteriormente, parece deberse a la diversidad bioquímica intraespecie de los lactobacilos vaginales y a la ausencia de algunas especies en las bases de datos.

Larsen y Monif (38) reportaron que estudios cuantitativos en lavados vaginales han encontrado 107 lactobacilos por g de secreción. Los LB más frecuentes son el L. acidophilus y el L. fermentum y 
los menos frecuentes o comunes son el L. plantarum, el L. brevis, el L. jensenii, el L. casei, el L. delbrueckii $y$ el L. salivarius. Rogosa y Sharpe (31) mencionan que más de una especie puede estar presente en una mujer.

\section{Homeostasis vaginal}

Las propiedades de la MBV que le permiten colonizar la mucosa e impedir el establecimiento o la proliferación excesiva de microorganismos potencialmente patógenos son de dos tipos: a) la adherencia específica a las células epiteliales y a los microorganismos patógenos, y b) la producción de compuestos antimicrobianos.

\section{a.- Adherencia específica}

La protección de la mucosa vaginal depende del reconocimiento específico entre las estructuras superficiales de los lactobacilos (adhesinas) y del epitelio (receptores).

Las adhesinas forman parte del glucocaliz de la pared celular y su composición parece ser variada, se han descrito los ácidos lipoproteicos (39), proteínas extracelulares (40), carbohidratos y glucoproteínas $(41,42)$. En relación con los receptores localizados en la superficie del epitelio no se conoce mucho, sin embargo, se ha encontrado que la fibronectina, que es una glucoproteína que forma parte de la matriz extracelular, es reconocida por los LB que han sido aislados en la vagina cuya unión se ve favorecida por el pH ácido de la vagina, así, hay cepas que no se unen a pH neutro como si lo hacen a pH ácido de 4 (43). Además de eso, la baja capacidad de unión de las bacterias intestinales anaeróbicas a la fibronectina (44) favorece a la estabilidad de la MBV. Igualmente, la unión de los lactobacilos podría ser de forma directa a los glucolípidos de la membrana de las células del epitelio vaginal en un proceso mediado por cationes divalentes
(41). El resultado de la asociación de los LB y el epitelio vaginal es la formación de una biopelícula que lo protege contra la colonización por microorganismos indeseados y patológicos (41); a este hecho se le agrega la capacidad que tienen los LB vaginales para coagregarse con los microorganismos potencialmente patológicos. La mayoría de las bacterias de la MBV tienen la propiedad de autoagregarse, propiedad que desaparece al adicionar la enzima proteinasa $\mathrm{K}$, por lo que esta propiedad depende de la producción de una proteína extracelular (41).

b.- Producción de compuestos antimicrobianos. Ácidos orgánicos (ácido láctico)

El pH fisiológico de la vagina es ácido con un rango de 3,5 a 4,5, con un valor medio de 3,5 \pm 0,2 y un promedio de 4; este $\mathrm{pH}$ ácido provoca un ambiente ácido en la vagina, el cual, es atribuible a la acumulación de $\operatorname{AL}(2,22)$, inhibe parcial o totalmente el desarrollo o crecimiento de la mayor parte de la bacterias provenientes del tracto digestivo y de las de origen ambiental, siendo esto, un mecanismo de protección natural de la mucosa vaginal muy eficaz (2). Como se ha mencionado anteriormente, el origen de la acidez vaginal es el AL que se genera como producto final del metabolismo fermentativo de los glúcidos, específicamente el glucógeno, liberado por la citólisis de las células superficiales e intermedias descamadas del epitelio vaginal, por parte de los lactobacilos. Esto ocurre desde la menarquia hasta la menopausia por lo que el glucógeno producido por las células del epitelio vaginal, es considerado la fuente principal que permite mantener el pH ácido de la vagina $(1,2)$; debido a esto, hay la creencia generalizada que los LB metabolizan el glucógeno a glucosa y esta a su vez, a AL, sin embargo, diferentes autores $(18,30,44,45)$ han reportado que el glucógeno producido por las células epiteliales de la vagina es degradado a glucosa por las propias células del epitelio vaginal, de manera que el papel de los LB sería solo la degradación de la glucosa a AL responsable de mantener la MBV y evitar la 


\section{J NÚÑEZ TROCONIS}

colonización de la vagina por parte de microorganismos indeseables. El apoyo de esta aseveración se basa en el hecho de encontrar los isómeros L (+) y D (-) del $\mathrm{AL}$ en cantidades similares al ácido. La formación o generación de estos isómeros es característica de los LB mientras que las células epiteliales generan el isómero L $(+)(46,47)$. Sin embargo, Boskey y cols. (47) sugirieron que el nivel protectivo vaginal del AL depende principalmente de la MBV pero las células de la vagina contribuyen entre un $4 \%$ al 30 $\%$ del total del AL producido en la vagina. Nasioudis y cols. (48) sugirieron que la cantidad de glucógeno disponible en la vagina por la exfoliación de la células superficiales e intermedias ricas en glucógeno se debe a la acción o actividad de la hialuronidadsa-1 y de la matrix de la metaloproteinasa (MMP), esto permite que la $\alpha$-amilasa degrade al glucógeno disponible en la vagina, el cual, es convertido en $\mathrm{AL} \mathrm{D(-)} \mathrm{por} \mathrm{los} \mathrm{LB}$, sin embargo, no se encontró ninguna correlación entre el isómero del AL L $(+)$ con la $\alpha$-amilasa, lo que sugiere que el isómero D (-) es el responsable de mantener el $\mathrm{pH}$ vaginal $\leq 4,5$ y prevenir el crecimiento de otras bacterias. Hearps y cols. (49) sugirieron que estos 2 isómeros del AL inducen una respuesta antiinflamatoria en las células humanas cervicovaginales contra el virus de la inmunodeficiencia adquirida (VIH); asimismo, Wagner y cols. (50) encontraron que los isómeros $\mathrm{D}(-)$ y $\mathrm{L}(+)$ del AL inhiben las deacetilasas, por lo tanto, incrementa la reparación del ADN, regulando la transcripción de los genes asociados a ellos. Diferentes autores $(23,51-53)$ han sugerido que el AL inhibe una amplia variedad de agentes infecciosos incluyendo a la Chlamydia trachomatis, el Herpes Virus Simple tipo 2 (HSV-2), el HIV, el HIV-1 y un rango amplio contra bacterias involucradas en la producción de la vaginitis bacteriana. Se ha demostrado que el AL afecta la respuesta inmunológica de la vagina a través de diferentes mecanismos tales como 1.- el aumento de la producción del mediador antiinflamatorio tales como el receptor antagonista de la interleuquina-1 (IL-1RA) por parte de las células del epitelio vaginal, 2.- la inhibición de la producción de mediadores proinflamatorios tales como IL-6, IL-8, el factor de necrosis tumoral-alfa $(\mathrm{TNF}-\alpha)$, el regulador de la activación de las células $\mathrm{T}$ expresadas y secretadas (RANTES), y la proteína-3 alfa del macrófago inflamatorio (MIP3 $\alpha$ ) (48); 3.- la liberación del factor transformador de crecimiento-beta (TGF- $\beta$ ) para estimular la respuesta antiviral (54); 4.- la estimulación de los T helper 17 (Th17) perteneciente a los linfocitos T, a través de la producción de la IL-23 al exponerse a los polisacáridos bacterianos (55); y 5.el aumento del AL a nivel citosólico, el cual bloquea la producción del monofosfato de adenosina cíclica (cAMP), lo que aumenta la capacidad de autofagia de las células epiteliales de la vagina para degradar los microorganismos y mantener la homeostasis (56). En general, estos estudios sugieren diferentes propiedades defensivas del AL más allá del papel o función de los isómeros. Estas propiedades separadas o juntas determinan la susceptibilidad del huésped y su relación con la MBV (1).

\section{Peróxido de hidrógeno}

El peróxido de hidrogeno $\left(\mathrm{H}_{2} \mathrm{O}_{2}\right)$ es otra substancia antimicrobiana producida a partir de la presencia del $\mathrm{O} 2$ y su producción parece ser común entre ciertas especies de LB como L. crispatus y L. jensenii, mientras que es excepcional en otras como L. fermentum y en los LB que habitan preferentemente en el intestino, como L. plantarum y L. casei, incluso aunque se aíslen de la vagina $(57,58)$. Los niveles de $\mathrm{O}_{2}$ en vagina son bajos con el objeto de mantener una atmósfera o medio anaeróbico (1). Las cepas productoras de $\mathrm{H}_{2} \mathrm{O}_{2}$ son más estables y suficientes en el ambiente vaginal (59) para proteger y mejorar la mucosa frente a las alteraciones causadas por microorganismos indeseados $(1,21,60$, 61 ), incluyendo aquellos productores de infecciones de transmisión sexual (ITS) como la Neisseria gonorrhoeae (62). El efecto antagonista o bactericida es mayor cuando se asocia a especies de LB tales como el L. crispatus o L. jensenii (58). Eschenbach y cols. 
(60) mencionaron que el $\mathrm{H}_{2} \mathrm{O}_{2}$ producido por los $\mathrm{LB}$ en la vagina pueden ser un mecanismo antimicrobiano no específico para mantener el ecosistema vaginal normal o sea la MBV. El efecto bactericida del $\mathrm{H}_{2} \mathrm{O}_{2}$ viene determinado por su capacidad oxidante y por la generación, a partir de ella, de metabolitos como el radical $\mathrm{OH}-$, que dañan la integridad del ADN celular. Dicho efecto se potencia por la mieloperoxidasa y los radicales haluros, como el $\mathrm{Cl}-$, que son abundantes en las secreciones biológicas y cuya concentración es elevada en el moco del cuello uterino, sobre todo durante la ovulación $(63,64)$. Boris y cols. (41) y Boris y Barbés (42), demostraron que el $\mathrm{H}_{2} \mathrm{O}_{2}$ producidos por los LB vaginales tiene un papel protector contra la Gv, suprimen el sobrecrecimiento de las candidas y la formación de las hifas. Sin embargo, el papel protector del $\mathrm{H}_{2} \mathrm{O}_{2}$ en la MBV es debatible (1).

\section{Bacteriocinas}

Son polipéptidos con actividad antimicrobiana que al contrario a los antibióticos peptídicos, se sintetizan en los ribosomas. Las bacterias lácticas producen multitud de bacteriocinas, alguna de las cuales, como la nisina, se emplean como conservante alimentario. Estas moléculas pueden producir la apertura de poros en las membranas e incluso la lisis celular, ya que algunas se unen al lípido II de la pared, este lípido es reconocido por la vancomicina $(65,66)$. Se han descrito múltiples actividades compatibles con la producción de bacteriocinas por los LB de origen vaginal pero solo hay dos en los que existe evidencia: uno de ellos es un péptido de 3,8 $\mathrm{kDa}$ activo sobre cepas de Gv, mientras que el otro inhibe diversas cepas de Enterococcus (67, 68). Las bacteriocinas incluyen a las bacteriocinas IIa, IIc, y J46, la acidocina IF221A, la gassericina $\mathrm{T}$ y la lantibiótica tipo-A, las cuales, son sustancias proteicas con actividad bactericida y sintetizadas o producidas por los LB en especial por el L. crispatus y el L. gasserei (69). Las bacterioricinas permeabilizan la membrana celular de los microrganismos patógenos tales como la del Staphylococcus aureus, Klebsiella spp., Eschericha fecalis y Eschericha coli, jugando un papel en la prevención del crecimiento de ellos (69). Sin embargo, el papel antagonista real de las bacteriocinas no se conoce bien, ya que su efecto bactericida solo se ha comprobado in vitro (2).

\section{Agentes tensioactivos}

Son compuestos anfifílicos, llamadas moléculas anfifílicas o antipáticas, originan o producen una disminución de la tensión superficial, favoreciendo la solubilización de sustancias hidrofóbicas. Se han descrito dos agentes tensioactivos, producidos uno por la cepa $L$. acidophilus y el otro por el $L$. fermentum, que inhiben la adhesión de E. faecalis y de E. coli, pero no la de $C$. albicans, a la goma de silicona de los catéteres urinarios $(70,71)$. Desafortunadamente, no se ha probado si también inhiben la adherencia de bacterias indeseadas a las células del epitelio vaginal. Por otra parte, los agentes tensioactivos solubilizan lípidos, de manera que podrían ser letales para virus envueltos y para micoplasmas, los cuales serían especialmente susceptibles al carecer de pared celular $(72,73)$.

\section{CONCLUSIÓN}

En resumen, desde hace casi 2 décadas, con las técnicas de microbiología molecular, se tiene una mejor idea de la complejidad de la MBV que ha permitido identificar o conocer bacterias no conocidas, las cuales, son importantes en la MBV. También, se ha aprendido que una sola bacteria no es el único componente importante de la MBV. Las técnicas de HTS han permitido tener una mejor visión de la diversidad bacteriana presente en el microambiente vaginal, así como también, ha permitido descubrir o develar la existencia de categorías de bacterias hasta entonces desconocidas. Esta revisión muestra los diferentes mecanismos que mantienen la salud u homeostasis de la vagina. 


\section{J NÚÑEZ TROCONIS}

\section{AGRADECIMIENTO}

Damos las gracias a la diseñadora gráfica Melissa Núñez de Ramírez por su colaboración y ayuda en la elaboración del gráfico presentado en este manuscrito

\section{Sin conflictos de interés.}

\section{REFERENCIAS}

1. Kalia N, Singh J, Kaur M. Microbiota in vaginal health and pathogenesis of recurrent vulvovaginal infections: a critical review. Ann Clin Microbiol Antimicrob. 2020; 19(1):5. doi: 10.1186/s12941-020-0347-4.

2. Martin DH. The microbiota of the vagina and its influence on women's health and disease. Am J Med Sci. 2012; 343(1):2-9. doi: 10.1097/MAJ.0b013e31823ea228.

3. Farage M, Maibach H. Lifetime changes in the vulva and vagina. Arch Gynecol Obstet. 2006; 273(4):195202. doi: 10.1007/s00404-005-0079-x.

4. Mendling W. Normal and abnormal vaginal microbiota J Lab Med 2016; 40(4):239-246. https://doi. org/10.1515/labmed-2016-0011

5. Linhares IM, Giraldo PC, Baracat EC. [New findings about vaginal bacterial flora]. Rev Assoc Med Bras (1992). 2010; 56(3):370-374. Portuguese. doi: 10.1590/ s0104-4230201

6. Dethlefsen L, McFall-Ngai M, Relman DA. An ecological and evolutionary perspective on humanmicrobe mutualism and disease. Nature. 2007; 449(7164):811-818. doi: 10.1038/nature06245.

7. Priestley CJ, Jones BM, Dhar J, Goodwin L. What is normal vaginal flora? Genitourin Med. 1997; 73(1):2328. doi: 10.1136/sti.73.1.23

8. Eschenbach DA, Patton DL, Hooton TM, Meier AS, Stapleton A, Aura J, et al. Effects of vaginal intercourse with and without a condom on vaginal flora and vaginal epithelium. J Infect Dis. 2001; 183(6):913-918. doi: 10 $.1086 / 319251$.

9. Eschenbach DA, Thwin SS, Patton DL, Hooton TM, Stapleton AE, Agnew K, et al. Influence of the normal menstrual cycle on vaginal tissue, discharge, and microflora. Clin Infect Dis. 2000; 30(6):901-907. doi: 10.1086/313818.

10. Gajer P, Brotman RM, Bai G, Sakamoto J, Schütte UM, Zhong $\mathrm{X}$, et al. Temporal dynamics of the human vaginal microbiota. Sci Transl Med. 2012; 4(132):132ra52. doi: 10.1126/scitranslmed.3003605.

11. Srinivasan S, Liu C, Mitchell CM, Fiedler TL, Thomas $\mathrm{KK}$, Agnew KJ, et al. Temporal variability of human vaginal bacteria and relationship with bacterial vaginosis. PLoS One. 2010; 5(4):e10197. doi: 10.1371/ journal.pone.0010197.

12. Li J, McCormick J, Bocking A, Reid G. Importance of vaginal microbes in reproductive health. Reprod Sci. 2012; 19(3):235-242. doi: 10.1177/1933719111418379.

13. Ravel J, Gajer P, Abdo Z, Schneider GM, Koenig SS, McCulle SL, et al. Vaginal microbiome of reproductiveage women. Proc Natl Acad Sci U S A. 2011; 108 Suppl 1(Suppl 1):4680-4687. doi: 10.1073/pnas.1002611107.

14. De Seta F, Campisciano G, Zanotta N, Ricci G, Comar $\mathrm{M}$. The vaginal community state types microbiomeimmune network as key factor for bacterial vaginosis and aerobic vaginitis. Front Microbiol. 2019; 10:2451. doi: 10.3389/fmicb.2019.02451.

15. MacNeill C, Umstead TM, Phelps DS, Lin Z, Floros $\mathrm{J}$, Shearer DA, et al. Surfactant protein A, an innate immune factor, is expressed in the vaginal mucosa and is present in vaginal lavage fluid. Immunology. 2004; 111(1):91-99. doi: 10.1111/j.1365-2567.2004.01782.x.

16. Nunn KL, Wang YY, Harit D, Humphrys MS, Ma B, Cone R, et al. Enhanced trapping of hiv-1 by human cervicovaginal mucus is associated with Lactobacillus crispatus-dominant microbiota. mBio. 2015; 6(5):e01084-15. doi: 10.1128/mBio.01084-15.

17. Arnold K, Birse K, Mckinnon L, Lingappa J, Novak $\mathrm{R}$, Westmacott $\mathrm{G}$, et al. Mucosal integrity factors are perturbed during bacterial vaginosis: a proteomic analysis. AIDS Res Hum Retroviru. 2014; 30(S1):A30. doi: 10.1089/aid.2014.5046.abstract.

18. Dominguez-Bello MG, Costello EK, Contreras M, Magris M, Hidalgo G, Fierer N, et al. Delivery mode shapes the acquisition and structure of the initial microbiota across multiple body habitats in newborns. Proc Natl Acad Sci U S A. 2010; 107(26):11971-11975. doi: 10.1073/pnas.1002601107.

19. Wylie JG, Henderson A. Identity and glycogenfermenting ability of lactobacilli isolated from the vagina of pregnant women. J Med Microbiol. 1969; 2(3):363-366. doi: 10.1099/00222615-2-3-363.

20. Bernbaum JC, Umbach DM, Ragan NB, Ballard JL, Archer JI, Schmidt-Davis H, et al. Pilot studies of estrogen-related physical findings in infants. Environ Health Perspect. 2008; 116(3):416-420. doi: 10.1289/ehp.10409. 


\section{MICROBIOTA DE LA VAGINA}

21. Alvarez-Olmos MI, Barousse MM, Rajan L, Van Der Pol BJ, Fortenberry D, Orr D, et al. Vaginal lactobacilli in adolescents: presence and relationship to local and systemic immunity, and to bacterial vaginosis. Sex Transm Dis. 2004; 31(7):393-400. doi: 10.1097/01. olq.0000130454.83883.e9.

22. Spear GT, French AL, Gilbert D, Zariffard MR, Mirmonsef P, Sullivan TH, et al. Human $\alpha$-amylase present in lower-genital-tract mucosal fluid processes glycogen to support vaginal colonization by Lactobacillus. J Infect Dis. 2014; 210(7):1019-1028. doi: 10.1093/infdis/jiu231.

23. O'Hanlon DE, Moench TR, Cone RA. Vaginal pH and microbicidal lactic acid when lactobacilli dominate the microbiota. PLoS One. 2013; 8(11):e80074. doi: 10.1371/journal.pone.0080074.

24. Keane FE, Ison CA, Taylor-Robinson D. A longitudinal study of the vaginal flora over a menstrual cycle. Int J STD AIDS. 1997; 8(8):489-494. doi: 10.1258/0956462971920631.

25. Schwebke JR, Richey CM, Weiss HL. Correlation of behaviors with microbiological changes in vaginal flora. J Infect Dis. 1999; 180(5):1632-1636. doi: $10.1086 / 315065$.

26. Weinberg ED. Iron availability and infection. Biochim Biophys Acta. 2009; 1790(7):600-605. doi: 10.1016/j. bbagen.2008.07.002.

27. Hickey RJ, Zhou X, Settles ML, Erb J, Malone K, Hansmann MA, et al. Vaginal microbiota of adolescent girls prior to the onset of menarche resemble those of reproductive-age women. mBio. 2015; 6(2):e00097-15. doi: 10.1128/mBio.00097-15.

28. Zhou X, Bent SJ, Schneider MG, Davis CC, Islam MR, Forney LJ. Characterization of vaginal microbial communities in adult healthy women using cultivationindependent methods. Microbiology (Reading). 2004; 150(Pt 8):2565-2573. doi: 10.1099/mic.0.26905-0.

29. Hyman R, Fukushima M, Diamond L, Kumm J, Giudice $\mathrm{L}$, Davis R. Microbes on the human vaginal epithelium. Proc Natl Acad Sci USA. 2005; 102:7952-7957. https:// doi.org/10.1073/pnas.0503236102.

30. Reid G, Burton J, Devillard E. The rationale for probiotics in female urogenital healthcare. MedGenMed [Internet]. 2004 [consultado 11 de marzo de 2021]; 6(1):49. Disponible en: https://www.ncbi.nlm.nih.gov/ pmc/articles/PMC1140735/
31. Rogosa M, Sharpe ME. Species differentiation of human vaginal lactobacilli. J Gen Microbiol. 1960; 23:197-201. doi: 10.1099/00221287-23-1-197.

32. Boyd MA, Antonio MA, Hillier SL. Comparison of API $50 \mathrm{CH}$ strips to whole-chromosomal DNA probes for identification of Lactobacillus species. J Clin Microbiol. 2005; 43(10):5309-5311. doi: 10.1128/ JCM.43.10.5309-5311.2005.

33. Song YL, Kato N, Matsumiya Y, Liu CX, Kato H, Watanabe K. Identification of Lactobacillus species of human origin by a commercial kit, API50CHL. Rinsho Biseibutshu Jinsoku Shindan Kenkyukai Shi. 1999; 10(2):77-82.

34. Song YL, Kato N, Matsumiya Y, Liu CX, Kato H, Watanabe K. Identification of and hydrogen peroxide production by fecal and vaginal lactobacilli isolated from Japanese women and newborn infants. J Clin Microbiol. 1999; 37(9):3062-3064. doi: 10.1128/ JCM.37.9.3062-3064.1999.

35. Vallor AC, Antonio MA, Hawes SE, Hillier SL. Factors associated with acquisition of, or persistent colonization by, vaginal lactobacilli: role of hydrogen peroxide production. J Infect Dis. 2001; 184(11):14311436. doi: 10.1086/324445.

36. Matsumiya Y, Kato N, Watanabe K, Kato H. Molecular epidemiological study of vertical transmission of vaginal Lactobacillus species from mothers to newborn infants in Japanese, by arbitrarily primed polymerase chain reaction. J Infect Chemother. 2002; 8(1):43-49. doi: 10.1007/s101560200005.

37. Pavlova SI, Kilic AO, Kilic SS, So JS, NaderMacias ME, Simoes JA, et al. Genetic diversity of vaginal lactobacilli from women in different countries based on 16S rRNA gene sequences. J Appl Microbiol. 2002; 92(3):451-459. doi: 10.1046/j.13652672.2002.01547.x.

38. Larsen B, Monif GR. Understanding the bacterial flora of the female genital tract. Clin Infect Dis. 2001; 32(4):e69-77. doi: 10.1086/318710.

39. Chan RC, Reid G, Irvin RT, Bruce AW, Costerton JW. Competitive exclusion of uropathogens from human uroepithelial cells by Lactobacillus whole cells and cell wall fragments. Infect Immun. 1985; 47(1):84-89. doi: 10.1128/iai.47.1.84-89.1985.

40. Reid G, Servin AL, Bruce AW, Busscher HJ. Adhesion of three Lactobacillus strains to human urinary and intestinal epithelial cells. Microbios. 1993; 75(302):57-65. 


\section{J NÚNEEZ TROCONIS}

41. Boris S, Suárez JE, Barbés C. Characterization of the aggregation promoting factor from Lactobacillus gasseri, a vaginal isolate. J Appl Microbiol. 1997; 83(4):413-420. doi: 10.1046/j.13652672.1997.00250.x.

42. Boris S, Barbés C. Role played by lactobacilli in controlling the population of vaginal pathogens. Microbes Infect. 2000; 2(5):543-546. doi: 10.1016/ s1286-4579(00)00313-0.

43. Nagy E, Fröman G, Mårdh PA. Fibronectin binding of Lactobacillus species isolated from women with and without bacterial vaginosis. J Med Microbiol. 1992; 37(1):38-42. doi: 10.1099/00222615-37-1-38.

44. Szöke I, Pascu C, Nagy E, Ljung A, Wadström T. Binding of extracellular matrix proteins to the surface of anaerobic bacteria. J Med Microbiol. 1996; 45(5):338343. doi: 10.1099/00222615-45-5-338.

45. Stewart-tull DE. Evidence that vaginal lactobacillo do not ferment glycogen. Am J Obstet Gynecol. 1964; 88:676-679. doi: 10.1016/0002-9378(64)90898-1.

46. Dellaglio F, De Roissart H, Torriani S, Curk MC, Janssens D. Caractéristiques générales des bactéries lactiques. En: de Roissart H, Luquet FM, editors. Bactéries Lactiques. Vol. I. Uriage: Lorica; 1994. p. 25116.

47. Boskey ER, Cone RA, Whaley KJ, Moench TR. Origins of vaginal acidity: high $\mathrm{D} / \mathrm{L}$ lactate ratio is consistent with bacteria being the primary source. Hum Reprod. 2001; 16(9):1809-1813. doi: 10.1093/ humrep/16.9.1809.

48. Nasioudis D, Beghini J, Bongiovanni AM, Giraldo PC, Linhares IM, Witkin SS. $\alpha$-Amylase in vaginal fluid: association with conditions favorable to dominance of Lactobacillus. Reprod Sci. 2015; 22(11):1393-1398. doi: 10.1177/1933719115581000.

49. Hearps AC, Tyssen D, Srbinovski D, Bayigga L, Diaz DJD, Aldunate $\mathrm{M}$, et al. Vaginal lactic acid elicits an anti-inflammatory response from human cervicovaginal epithelial cells and inhibits production of pro-inflammatory mediators associated with HIV acquisition. Mucosal Immunol. 2017; 10(6):14801490. doi: 10.1038/mi.2017.27.

50. Wagner W, Ciszewski WM, Kania KD. L- and D-lactate enhance DNA repair and modulate the resistance of cervical carcinoma cells to anticancer drugs via histone deacetylase inhibition and hydroxycarboxylic acid receptor 1 activation. Cell Commun Signal. 2015; 13:36. doi: 10.1186/s12964-015-0114-x.
51. Ñahui Palomino RA, Zicari S, Vanpouille C, Vitali B, Margolis L. Vaginal Lactobacillus Inhibits HIV-1 Replication in Human Tissues Ex Vivo. Front Microbiol. 2017; 8:906. doi: 10.3389/fmicb.2017.00906.

52. Isaacs $\mathrm{CE}, \mathrm{Xu} \mathrm{W}$. Theaflavin-3,3'-digallate and lactic acid combinations reduce herpes simplex virus infectivity. Antimicrob Agents Chemother. 2013; 57(8):3806-3814. doi: 10.1128/AAC.00659-13.

53. Gong Z, Luna Y, Yu P, Fan H. Lactobacilli inactivate Chlamydia trachomatis through lactic acid but not $\mathrm{H}_{2} \mathrm{O}$ 2. PLoS One. 2014; 9(9):e107758. doi: 10.1371/ journal.pone.0107758.

54. Mossop H, Linhares IM, Bongiovanni AM, Ledger WJ, Witkin SS. Influence of lactic acid on endogenous and viral RNA-induced immune mediator production by vaginal epithelial cells. Obstet Gynecol. 2011; 118(4):840-846. doi: 10.1097/ AOG.0b013e31822da9e9.

55. Witkin SS, Alvi S, Bongiovanni AM, Linhares IM, Ledger WJ. Lactic acid stimulates interleukin-23 production by peripheral blood mononuclear cells exposed to bacterial lipopolysaccharide. FEMS Immunol Med Microbiol. 2011; 61(2):153-158. doi: 10.1111/j.1574-695X.2010.00757.x.

56. Ghadimi D, de Vrese M, Heller KJ, Schrezenmeir J. Lactic acid bacteria enhance autophagic ability of mononuclear phagocytes by increasing Th1 autophagypromoting cytokine (IFN-gamma) and nitric oxide (NO) levels and reducing Th2 autophagy-restraining cytokines (IL-4 and IL-13) in response to Mycobacterium tuberculosis antigen. Int Immunopharmacol. 2010; 10(6):694-706. doi: 10.1016/j.intimp.2010.03.014.

57. Song YL, Kato N, Matsumiya Y, Liu CX, Kato H, Watanabe K. Identification of and hydrogen peroxide production by fecal and vaginal lactobacilli isolated from Japanese women and newborn infants. J Clin Microbiol. 1999; 37(9):3062-3064. doi: 10.1128/ JCM.37.9.3062-3064.1999.

58. Antonio MA, Rabe LK, Hillier SL. Colonization of the rectum by Lactobacillus species and decreased risk of bacterial vaginosis. J Infect Dis. 2005; 192(3):394-398. doi: $10.1086 / 430926$.

59. Vallor AC, Antonio MA, Hawes SE, Hillier SL. Factors associated with acquisition of, or persistent colonization by, vaginal lactobacilli: role of hydrogen peroxide production. J Infect Dis. 2001; 184(11):14311436. doi: $10.1086 / 324445$. 
60. Eschenbach DA, Davick PR, Williams BL, Klebanoff SJ, Young-Smith K, Critchlow CM, et al. Prevalence of hydrogen peroxide-producing Lactobacillus species in normal women and women with bacterial vaginosis. J Clin Microbiol. 1989; 27(2):251-256. doi: 10.1128/ JCM.27.2.251-256.1989.

61. Hawes SE, Hillier SL, Benedetti J, Stevens CE, Koutsky LA, Wolner-Hanssen $\mathrm{P}$, et al. Hydrogen peroxide-producing lactobacilli and acquisition of vaginal infections. J Infect Dis. 1996; 174(5):10581063. doi: 10.1093/infdis/174.5.1058.

62. St Amant DC, Valentin-Bon IE, Jerse AE. Inhibition of Neisseria gonorrhoeae by Lactobacillus species that are commonly isolated from the female genital tract. Infect Immun. 2002; 70(12):7169-7171. doi: 10.1128/ iai.70.12.7169-7171.2002.

63. Klebanoff SJ, Belding ME. Virucidal activity of $\mathrm{H}_{2} \mathrm{O}_{2}-$ generating bacteria: requirement for peroxidase and a halide. J Infect Dis. 1974; 129(3):345-348. doi: 10.1093/infdis/129.3.345.

64. Klebanoff SJ, Waltersdorph AM. Prooxidant activity of transferrin and lactoferrin. J Exp Med. 1990; 172(5):1293-1303. doi: 10.1084/jem.172.5.1293.

65. Nes IF, Diep DB, Håvarstein LS, Brurberg MB, Eijsink $\mathrm{V}$, Holo H. Biosynthesis of bacteriocins in lactic acid bacteria. Antonie Van Leeuwenhoek. 1996; 70(24):113-128. doi: 10.1007/BF00395929.

66. Wiedemann I, Böttiger T, Bonelli RR, Schneider T, Sahl HG, Martínez B. Lipid II-based antimicrobial activity of the antibiotic plantaricin C. Appl Environ Microbiol. 2006; 72(4):2809-2814. doi: 10.1128/AEM.72.4.28092814.2006.

67. Aroutcheva AA, Simoes JA, Faro S. Antimicrobial protein produced by vaginal Lactobacillus acidophilus that inhibits Gardnerella vaginalis. Infect Dis Obstet Gynecol. 2001;9(1):33-39. doi: 10.1155/ S1064744901000060.
68. Ocaña VS, Pesce De Ruiz Holgado AA, Nader-Macías ME. Characterization of a bacteriocin-like substance produced by a vaginal Lactobacillus salivarius strain. Appl Environ Microbiol. 1999; 65(12):5631-5635. doi: 10.1128/AEM.65.12.5631-5635.1999.

69. Stoyancheva G, Marzotto M, Dellaglio F, Torriani S. Bacteriocin production and gene sequencing analysis from vaginal Lactobacillus strains. Arch Microbiol. 2014; 196(9):645-653. doi: 10.1007/s00203-014-10031.

70. Velraeds MM, van de Belt-Gritter B, van der Mei HC, Reid G, Busscher HJ. Interference in initial adhesion of uropathogenic bacteria and yeasts to silicone rubber by a Lactobacillus acidophilus biosurfactant. J Med Microbiol. 1998; 47(12):1081-1085. doi: 10.1099/00222615-47-12-1081.

71. Velraeds MM2, van de Belt-Gritter B, Busscher HJ, Reid G, van der Mei HC. Inhibition of uropathogenic biofilm growth on silicone rubber in human urine by lactobacilli--a teleologic approach. World J Urol. 2000; 18(6):422-426. doi: 10.1007/p100007084.

72. Vollenbroich D, Ozel M, Vater J, Kamp RM, Pauli G. Mechanism of inactivation of enveloped viruses by the biosurfactant surfactin from Bacillus subtilis. Biologicals. 1997; 25(3):289-297. doi: 10.1006/ biol.1997.0099.

73. Vollenbroich D 2, Pauli G, Ozel M, Vater J. Antimycoplasma properties and application in cell culture of surfactin, a lipopeptide antibiotic from Bacillus subtilis. Appl Environ Microbiol. 1997; 63(1):44-49. doi: 10.1128/AEM.63.1.44-49.1997.

Recibido 29 de julio de 2021 Aprobado 26 de octubre de 2021 